\title{
ON SEMIABELIAN GROUPS
}

\author{
N. F. KUZENNYI AND I. YA. SUBBOTIN
}

Received 21 June 2004 and in revised form 25 August 2004

We establish some structural results on the semiabelian groups, that is, groups generated by their cyclic normal subgroups. Such groups play a significant role in the theory of supersoluble groups. A description of two-generated semiabelian groups and torsion-free semiabelian groups is obtained.

Recall that a group is called semiabelian if it is generated by its normal cyclic subgroups [6]. The class of semiabelian groups is a very natural generalization of the wellknown class of Dedekind groups (the groups in which all cyclic subgroups are normal). In the paper [6] Venzke showed that these groups could play a major role in the theory of supersoluble finite groups. Based on the notion of semiabelian group, he developed a theory of finite supersoluble group similar to the theory of finite nilpotent group. He also proved that finite semiabelian groups are nilpotent of class 2, closed under homomorphic images and direct products, and are not closed under subgroups or normal subgroups. In this paper we study semiabelian (finite and infinite) groups under some restrictions. In particular, the torsion-free semiablian groups and two-generated semiabelian groups are described.

Lemma 1. (i) Any semiabelian group can be presented as a product of normal infinite and normal cyclic p-subgroup.

(ii) Any factor group of a direct product of semiabelian groups is semiabelian.

(iii) There are semiabelian groups with non-semiabelian normal subgroups.

Proof. The first and the second assertions are obvious (see also [6, page 573]). In [6, page 573] one can find an appropriate example of a semiabelian $p$-group for odd prime $p$, which contains a non-semiabelian normal subgroup.

Lemma 2. A derived subgroup $[G, G]$ of a semiabelian group $G$ is periodic and central. It coincides with the derived subgroup $[H, H]$ of a periodic subgroup $H$ decomposing into a product of G-normal cyclic subgroups. Moreover, $G=H Z$, where $Z \leq Z(G)$ is a subgroup generated by infinite normal in $G$ cyclic subgroups. 
Proof. By Lemma $1, G=\prod_{i \in I}\left\langle g_{i}\right\rangle$ (here and below $\prod_{i \in I} K_{i}$ is a product of subgroups $K_{i}$, $i \in I$ ), where $\left\langle g_{i}\right\rangle \unlhd G$ for all $i \in I$. Denote by $G_{i}=C_{G}\left(g_{i}\right)$ the centralizer of element $g_{i}$ in $G, i \in I$. Then $G_{i} \unlhd G$, and $G / G_{i} \leq \operatorname{Aut}\left(\left\langle g_{i}\right\rangle\right)$ is abelian as a group of automorphism of a cyclic group. Hence, $[G, G] \leq G_{i}$. It is clear that $\bigcap_{i \in I} G_{i}=Z(G)$ and $[G, G] \leq Z(G)$.

It is obvious that in a group with the property $[G, G] \leq Z(G)$, for any two elements $a, b \in G$ the following equation holds: $[a, b]^{n}=\left[a, b^{n}\right]$ (see [5, Exercise 5.1.4]). This fact will help us to show that $[G, G]$ is a periodic group. In turn, $[G, G]$ is generated by commutators $\left[g_{i}, g_{j}\right]$. Assume that one of the elements $g_{i}$ or $g_{j}$ (let say $g_{i}$ ) has an infinite order. If $\left|g_{j}\right|=n$, then $\left[g_{i}, g_{j}\right]^{n}=\left[g_{i}, g_{j}^{n}\right]=1$. So $\left|\left[g_{i}, g_{j}\right]\right|<\infty$. Therefore, we need to consider the case when $\left|g_{j}\right|=\left|g_{i}\right|=\infty$. If $\left\langle g_{i}\right\rangle \cap\left\langle g_{j}\right\rangle=1$, then $\left[g_{i}, g_{j}\right]=1$. If $\left\langle g_{i}\right\rangle \cap\left\langle g_{j}\right\rangle \neq 1$, then $\left\langle g_{i}\right\rangle \cap\left\langle g_{j}\right\rangle=\left\langle g_{j}^{n}\right\rangle \leq Z\left(\left\langle g_{i}, g_{j}\right\rangle\right)$. In this case, the commutator identity $[a, b]^{n}=\left[a, b^{n}\right]$ implies that $1=\left[g_{i}, g_{j}^{n}\right]=\left[g_{i}, g_{j}\right]^{n}$. Hence, $[G, G]$ is generated by elements of finite order. Since it is central, $[G, G]$ is periodic.

Denote by $Z$ the product of all $\left\langle g_{i}\right\rangle$ of infinite order. Since $[G, G]$ is periodic, $[G, G] \cap$ $\left\langle g_{i}\right\rangle=1$ for any $\left\langle g_{i}\right\rangle$ of infinite order. Therefore, $\left\langle g_{i}\right\rangle \leq Z(G)$, and hence $Z \leq Z(G)$. Let $H$ be the product of all $\left\langle g_{j}\right\rangle$ such that $\left\langle g_{j}\right\rangle \not Z Z(G)$. Then such $\left\langle g_{j}\right\rangle$ have finite orders, $H$ is periodic, and $G=H Z$. Since $Z \leq Z(G),[G, G]=[H, H]$.

The following corollary has been proven above.

Corollary 3. If a group $G$ decomposes into a product of its normal cyclic subgroups $\left\langle g_{i}\right\rangle$, then every infinite factor $\left\langle g_{i}\right\rangle$ is central.

Corollary 4. A finitely generated semiabelian group $G$ decomposes as a direct product of a finite number of factors, each of which is either a finitely generated abelian group or a finite semiabelian Sylow p-subgroup of $G$.

Proof. Let $G$ be a finitely generated semiabelian group. By Lemma 2, $G=H Z$, where $Z \leq Z(G), H$ is periodic, and $[H, H]=[G, G] \leq Z(G)$. Since $G$ is a nilpotent finitely generated group, its periodic part $t(G)$ is also finitely generated (see, e.g., [5, 5.2.17]). Therefore, $H$ is finite. Let $C=t(G) \cap Z$. It is obvious that $G=t(G) Z$, and $C=t(Z)$. Since $G$ is finitely generated, the factor group $G / t(G)$ is also finitely generated, and therefore by Lemmas 1 and 2 it is abelian. Then $Z / t(Z)$ is a finitely generated abelian group. By a Kulikov theorem (see, e.g., [3, page 174]), $Z=C \times D$, where $D$ is a finitely generated abelian group. Since $D$ is a central subgroup, $G=t(G) \times D$. Obviously, $t(G)=H C$. Lemma 2 implies that $H$ decomposes into a product of $G$-invariant cyclic subgroups. Since $C \leq Z(G)$, it also has this property. Therefore, $t(G)$ also decomposes into a product of $G$-normal cyclic subgroups. In [6, Theorem 2.1] Venzke proved that a semiabelian finite group is nilpotent of class 2, and each Sylow p-subgroup of such a group is also semiabelian.

Lemma 2 implies the following theorem.

Theorem 5. Semiabelian torsion free groups are abelian.

Corollary 6. A nomperiodic semiabelian two-generated group decomposes into a direct product of two of its cyclic subgroups. 
Proof. Let $G$ be a nonperiodic two-generated semiabelian group. By Corollary $4, G=$ $t(G) \times D$, where $D$ an abelian finitely generated group. Since $G$ is two-generated nonperiodic, $D$ is at most two-generated, and $D \neq 1$. If rank of $D$ is 2 , then $t(G)=1$, and everything is proved. If $D$ is a cyclic, then $t(G)$ is also cyclic, and our corollary is proved.

A group $G$ is called the almost direct product of groups $G_{i}, i \in I$, if it satisfies the following conditions:

(1) for any $G_{i}$ group $G$ contains a normal subgroup $G_{i}^{*} \cong G_{i}$;

(2) $G=\prod_{i \in I} G_{i}^{*}$;

(3) for any $i \in I G_{i}^{*} \cap \prod_{i, j, \in I, j \neq i} G_{j}^{*}<G_{i}^{*}$.

We say that $G_{i}^{*}$ is an almost direct factor of the corresponding almost direct decomposition of $G$, and the cardinality $|I|$ is the cardinality of this almost direct product.

An almost direct product is a wide generalization of direct and central products (see, e.g., [2]). However, not every group being a product of its, let say, cyclic subgroups decomposes into an almost direct product of its cyclic subgroups. For example, every Dedekind group is a product of its cyclic subgroups, but not every abelian group (a quasicyclic group, an additive group of rational numbers) is an almost direct product of its cyclic subgroups.

Lemma 7. Let $G$ be a semiabelian p-group of bounded exponent. Then $G /[G, G]$ is a direct product of cardinality $\sigma$ of its cyclic p-subgroups, and $G$ is an almost direct product of cardinality $\sigma$ of its normal cyclic subgroups.

Proof. By Lemma $1 G$ is a product of its normal subgroups $\left\langle g_{i}\right\rangle, i \in I$. Since the exponent of $G$ is bounded, then $G /[G, G]$ is a direct product of its cyclic subgroups $\left\langle[G, G] a_{l}\right\rangle, l \in L$, such that $\left|\left\langle[G, G] a_{l}\right\rangle\right|>1$. Let $\Phi /[G, G]$ denote the Frattini subgroup $\Phi(G /[G, G])$ of the group $G /[G, G]$. Then $\Phi /[G, G]$ is a direct product of subgroups $\left\langle[G, G] a_{l}^{p}\right\rangle, l \in L$. It is clear that $\Phi \unlhd G$, and $G / \Phi$ is a direct product of subgroups $\left\langle\Phi a_{l}\right\rangle,\left|\Phi a_{l}\right|=p, l \in L$. Hence, $G / \Phi$ is an elementary abelian $p$-group decomposed into a direct product of cardinality $|L|$ of cyclic subgroups of prime order $p$. Clearly, $G / \Phi$ is generated by cosets $\Phi g_{i}, i \in I$, such that $\left|\Phi g_{i}\right| \leq p$. Then $I$ contains a subset $I_{1}$ such that for any $i \in I_{1}\left|\Phi g_{i}\right|=p$, and $G / \Phi$ is a direct product of such factors $\left\langle\Phi g_{i}\right\rangle$. By the theorem about the invariants of a decomposition of abelian $p$-groups into a direct product of cyclic subgroups (see, e.g., [3, page 147]) $|L|=\left|I_{i}\right|$, and without loss of generality, we can assume that $L=I_{1}$. Let $N \leq G$ be a product of $\left\langle g_{i}\right\rangle, i \in L$. Clearly, $N \unlhd G$. Put $X=[G, G] N$. Obviously, $X \unlhd G$. Since $G / \Phi$ is a direct product of subgroups $\left\langle\Phi g_{i}\right\rangle, i \in I$, we can conclude that $G=\Phi N=\Phi X$. Put $Y=\Phi \cap X$. Then $[G, G] \leq Y \unlhd G, G / Y=\Phi / Y \times X / Y$. Since $[G, G] \leq Y$ and $\Phi([G, G])=$ $\Phi(G /[G, G])$, we can write

$$
G / Y \cong(G /[G, G]) /(Y /[G, G])=(\Phi /[G, G]) /(Y /[G, G]) \times(X /[G, G]) /(Y /[G, G]) .
$$

Since $G /[G, G]$ is a direct product of cyclic groups, and $\exp (G /[G, G])$ is bounded, it follows that the Frattini subgroup of any homomorphic image of $G /[G, G]$ coincides with the homomorphic image of the Frattini subgroup of the group, that is, $\Phi / Y=\Phi(G / Y)$. Since the exponent of $G / Y$ is bounded, in the case when $|\Phi / Y|>1$, there is a maximal subgroup in $G / Y$, which does not include $\Phi / Y$, a contradiction. Hence, $\Phi / Y=1, \Phi \leq X$, 
and $G=X=[G, G] N$. By Lemma 2, $[G, G]$ is abelian, and therefore $N=G$. Then $G$ is a product of $\left\langle g_{i}\right\rangle, i \in L$. So $G / \Phi$ decomposes into a direct product of $\left\langle\Phi g_{i}\right\rangle,\left|\Phi g_{i}\right|=p$.

Let $i \neq j$, and let $G_{i}$ be the product of all $\left\langle g_{j}\right\rangle, j \in L$. Clearly, $G_{i} \unlhd G$, and $\left|G: G_{i}\right|<\infty$. Put $G_{i}^{*}=\left\langle g_{i}^{p}\right\rangle G_{i}$. Then $\left|G: G_{i}^{*}\right| \leq p$. It follows that $[G, G] \leq G_{i}^{*}, \Phi \leq G_{i}^{*}$, and $G_{i}^{*} \unlhd$ $G$. Consider the group $G / \Phi$. It is a direct product of $\left\langle\Phi g_{i}\right\rangle, i \in L$, and $G_{i}^{*} / \Phi$ is a direct product of all $\left\langle\Phi g_{j}\right\rangle, j \in L, i \neq j$. It follows that $G_{i}^{*} \cap\left\langle g_{i}\right\rangle=\left\langle g_{i}\right\rangle$, and $\left\langle g_{i}\right\rangle \cap G_{i} \leq\left\langle g_{i}\right\rangle$.

Using the results above, we obtain the following theorem describing the two-generated semiabelain groups.

THeOREM 8. Two-generated semiabelian groups are exhausted by the following types of groups:

(1) $G=\langle a\rangle \times\langle b\rangle$;

(2) $G=\langle a, b\rangle$, G is a quaternion group, $|a|=|b|=4,[a, b]=a^{2}=b^{2}$;

(3) $G=\langle a\rangle \lambda\langle b\rangle,|a|=p^{\alpha},|b|=p^{\beta},\langle[a, b]\rangle=\left\langle a^{p^{k}}\right\rangle, \alpha \geq k \geq \alpha-k>0, p^{k}>2, k \geq$ $\beta \geq \alpha-k$

(4) $G$ is a finite nilpotent nonabelian non-p-group, every Sylow p-subgroup of which is a group of one of the types 1-3 above or cyclic.

Proof. Necessity. Observe that if $G$ is a nonperiodic group, then by Corollary 6, $G$ is a direct product of two cyclic groups; that is, $G$ is a group of the type 1. By Lemma 2, we can assume that $G$ is a periodic two-generated nilpotent group; therefore $G$ is finite, and $G$ is a direct product of its Sylow $p$-subgroups. If $G$ is abelian, then it is a group of the type 1 . If $G$ is nonabelian, then it includes a nonabelian Sylow $p$-subgroup $P$ whose minimal number of generators is 2. Further we consider two cases: $G=P$, and $G$ is a non- $p$ nonabelian group. In the first case $G$ is a metacyclic (in the meaning of [5, page 290]) two-generated p-group. Since $P$ is nonabelian, and $[G, G] \leq \Phi(G)$ (the Frattini subgroup of $G$ ), $G /[G, G]$ is a direct product of two cyclic subgroups. By Lemma 7, $G$ is an almost direct product of two cyclic subgroups $\left\langle g_{1}\right\rangle$ and $\left\langle g_{2}\right\rangle$. Assume that $\left|g_{1}\right| \geq\left|g_{2}\right|$, and denote $\left\langle g_{1}\right\rangle=\langle a\rangle$. Since $G=\left\langle g_{1}, g_{2}\right\rangle,\left\langle g_{1}\right\rangle \triangleleft G$ and $\left\langle g_{2}\right\rangle \triangleleft G$, we can observe that $[G, G] \leq\left\langle g_{1}\right\rangle \cap\left\langle g_{2}\right\rangle \leq Z(G)$. $G$ is nonabelian, and therefore $a^{p^{\alpha}}=1$ some $\alpha \geq 2$.

If $|a|=4$ and $\left|g_{2}\right|=2$, then $G=\left\langle a, g_{2}\right\rangle$ is an abelian group, which is impossible. So $\left|g_{2}\right|>2$, that is, $\left|g_{1}\right|=\left|g_{2}\right|=4$, and $G$ belongs to the type 2 .

If $\left.|a|>4,\left[\langle a\rangle,\left\langle g_{2}\right\rangle\right]=\left\langle a^{p^{k}}\right\rangle, \alpha\right\rangle k, p^{k}>2$. Since $G$ is a nonabelian nilpotent group with a central derived subgroup, and $G=\langle a\rangle\left\langle g_{2}\right\rangle,|a| \geq\left|g_{2}\right|,|a|>4,\left[\langle a\rangle,\left\langle g_{2}\right\rangle\right]=\left\langle a p^{k}\right\rangle$, $\alpha>k, p^{k}>2$, using the detailed classification of metacyclic $p$-groups given in [4, Theorem 1.2.2], we obtain that $\left.G=\langle a\rangle \lambda\langle b\rangle,|a|=p^{\alpha},|b|=p^{\beta},\langle[a, b]\rangle=\left\langle a^{p^{k}}\right\rangle, k \geq \alpha-k\right\rangle 0$, $\alpha \geq \beta \geq \alpha-k$. Obviously, we can write $g_{2}=a^{i} b^{j}$ for some whole numbers $i, j$, and since $G=\langle a\rangle\left\langle g_{2}\right\rangle,(j, p)=1$. Since $\left\langle g_{2}\right\rangle \geq[G, G]=\left\langle a^{p^{k}}\right\rangle \leq Z(G)$, it is follows that there is a positive whole number $\delta$ such that $\left\langle g_{2}^{p^{\delta}}\right\rangle=\left\langle a^{p^{k}}\right\rangle$. Using [4, Proposition 1.1.10 (2)] (see also the formula obtained inductively in the proof of [1, Theorem 12.5.4]), we can write

$$
g_{2}^{p^{\delta}}=\left(a^{i} b^{j}\right)^{p^{\delta}}=a^{i p^{\delta}} b^{j p^{\delta}}[a, b]^{-(1 / 2) i j p^{\delta}\left(p^{\delta}-1\right)}
$$


and $\left\langle g_{2}^{p^{\delta}}\right\rangle \leq\left\langle a^{p^{k}}\right\rangle$. It is clear that $b^{j p^{\delta}}=1,[a, b]=a^{l p^{k}},(l, p)=1$. Therefore,

$$
[a, b]^{-(1 / 2) i j p^{\delta}\left(p^{\delta}-1\right)}=a^{-(1 / 2) l i j p^{\delta+k}\left(p^{\delta}-1\right)} .
$$

Hence,

$$
g_{2}^{p^{\delta}}=a^{i p^{\delta}} a^{-(1 / 2) l i j p^{\delta+k}\left(p^{\delta}-1\right)}
$$

Since $p^{k}>2,-(1 / 2) l i j p^{\delta+k}\left(p^{\delta}-1\right)=t p^{\delta+1}$ for some whole number $t$. Then $a^{i p^{\delta}} a^{t p^{\delta+1}}$ $=a^{p^{\delta}(i+t p)}$. Since $g_{2}^{p^{\delta}} \leq\left\langle a^{p^{k}}\right\rangle$ and $\left\langle g_{2}^{p^{\delta}}\right\rangle=\left\langle a^{p^{\delta}(i+t p)}\right\rangle$, we can conclude that $\delta \leq k$. Since $\delta \geq \beta, k \geq \beta$, and $G$ is a group from the type 3 .

In the second case when $G$ is not a $p$-group, it is a direct product of its Sylow $p_{i}{ }^{-}$ subgroups, each of which by Corollary 4 is a semiabelian two-generated group or a cyclic group. By Lemma 7 each of these Sylow subgroups is a product of no more than two normal in $G$ cyclic subgroups.

Sufficiency. For the groups of types 1 and 2, the sufficiency is obvious.

For the type $\left.3, G=\langle a\rangle \lambda\langle b\rangle,|a|=p^{\alpha},|b|=p^{\beta},\langle[a, b]\rangle=\left\langle a^{p^{k}}\right\rangle, \alpha \geq k \geq \alpha-k\right\rangle 0$, $p^{k}>2, k \geq \beta \geq \alpha-k,[4$, Theorem 1.2.2] implies that for such metacyclic groups $[G, G] \leq$ $Z(G)$. Using the formula from the proof of [1, Theorem 12.5.4] (see, also, [4, Proposition $1.1 .10(2)])$, we can obtain for $g=a b$,

$$
g^{p^{\beta}}=a^{p^{\beta}} b^{p^{\beta}}[a, b]^{-(1 / 2) p^{\beta}\left(p^{\beta}-1\right)}=a^{p^{\beta}}\left(a^{l p^{k}}\right)^{-(1 / 2) p^{\beta}\left(p^{\beta}-1\right)}=a^{p^{\beta}} a^{-(1 / 2) l p^{\beta+k}\left(p^{\beta}-1\right)},
$$

where $(l, p)=1$. Since $p^{k}>2$, we can find a number $t$, such that $-(1 / 2) l p^{\beta+k}\left(p^{\beta}-1\right)=$ $t p^{\beta+1}$, and therefore $g p^{p^{\beta}}=a^{p^{\beta}(1+t p)}$, where $p \nmid 1+t p$. By the conditions, $\beta \leq k\left\langle g p^{p^{\beta}}\right\rangle \geq$ $\left\langle a^{p^{k}}\right\rangle=[G, G]$. Therefore, $\langle g\rangle \triangleleft G$, and $G=\langle a, b\rangle=\langle a, a b\rangle=\langle a, g\rangle$. Hence, $G=\langle a\rangle\langle g\rangle$ is a semiabelian two-generated group.

For the groups of type 4, the sufficiency directly follows from the sufficiency of the types $1-3$.

\section{References}

[1] M. Hall Jr., The Theory of Groups, Macmillan, New York, 1959.

[2] B. Huppert, Endliche Gruppen. I, Die Grundlehren der Mathematischen Wissenschaften, vol. 134, Springer, New York, 1967.

[3] A. G. Kurosh, Group Theory, Nauka, Moscow, 1967.

[4] N. F. Kuzennyi and N. N. Semko, Metahamiltonian Groups and Their Generalizations, National Academy of Sciences, Kiev, 1996.

[5] D. J. S. Robinson, A Course in the Theory of Groups, Graduate Texts in Mathematics, vol. 80, Springer, New York, 1996. 


\section{On semiabelian groups}

[6] P. Venzke, A contribution to the theory of finite supersolvable groups, J. Algebra 57 (1979), no. 2, $567-579$.

N. F. Kuzennyi: Algebra Department, Institute of Mathematics, National Academy of Science, 3 Tereshenkivska Street, 01601 Kyiv-4, Ukraine

E-mail address: kuzen@imath.kiev.ua

I. Ya. Subbotin: Department of Mathematics, National University, 9920 South La Cienega Boulevard, Inglewood, CA 90301, USA

E-mail address: isubboti@nu.edu 


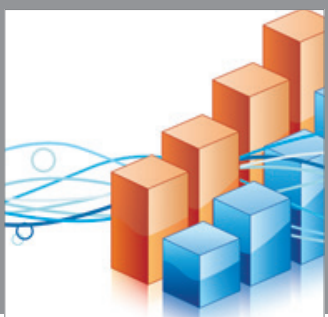

Advances in

Operations Research

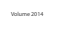

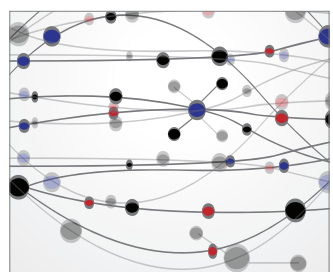

\section{The Scientific} World Journal
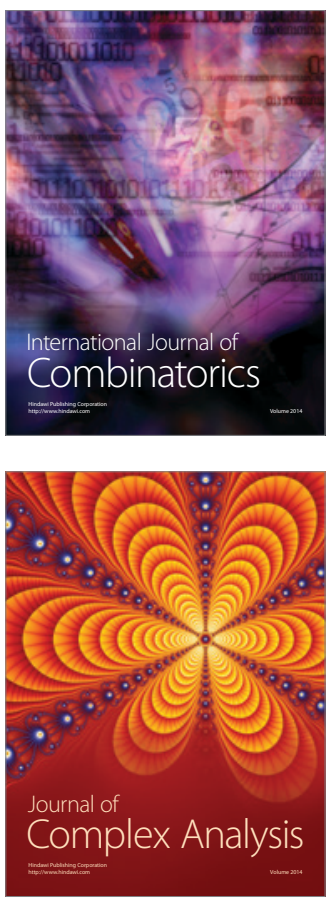

International Journal of

Mathematics and

Mathematical

Sciences
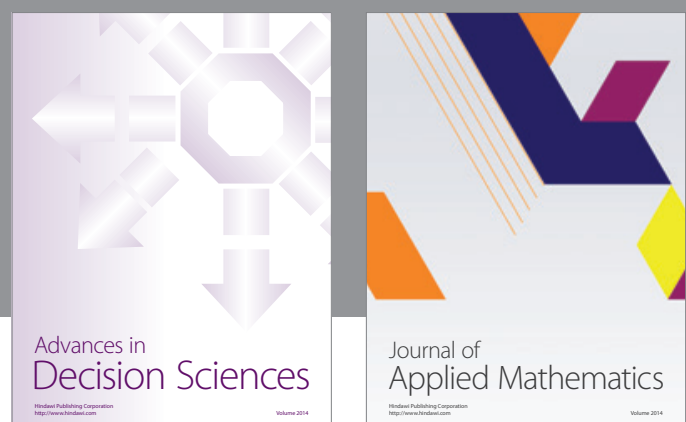

Journal of

Applied Mathematics
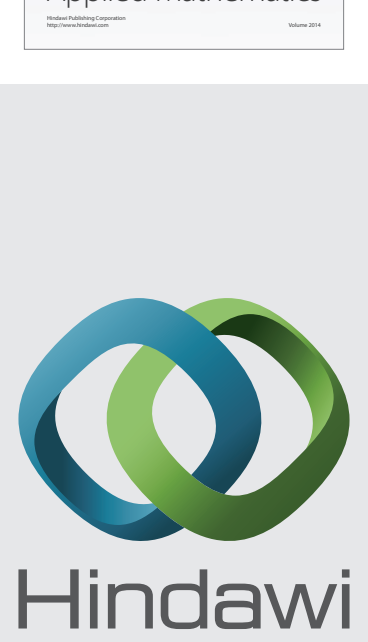

Submit your manuscripts at http://www.hindawi.com
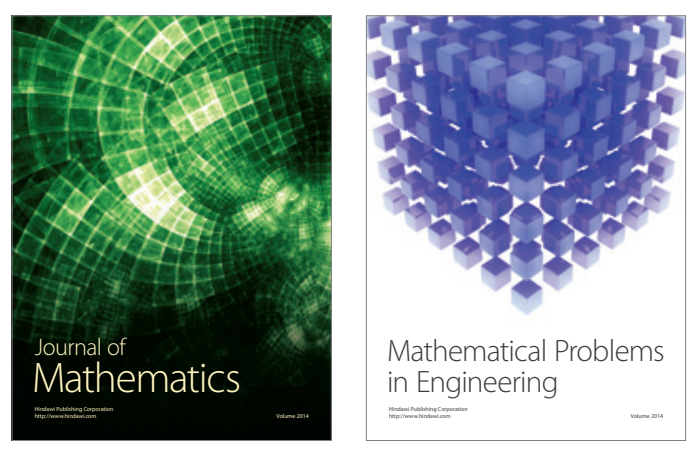

Mathematical Problems in Engineering
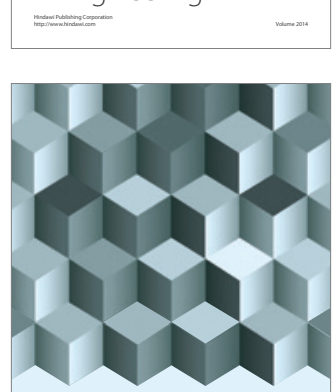

Journal of

Function Spaces
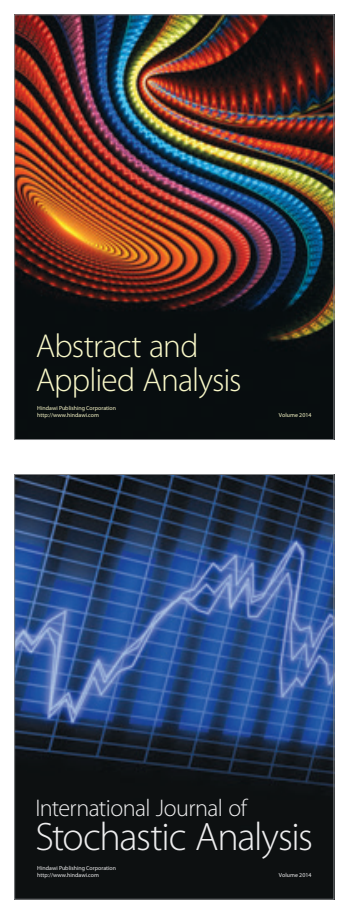

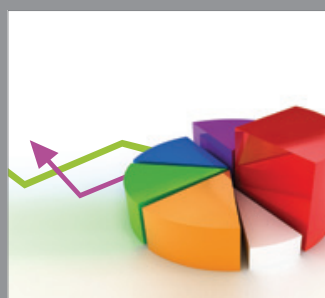

ournal of

Probability and Statistics

Promensencen
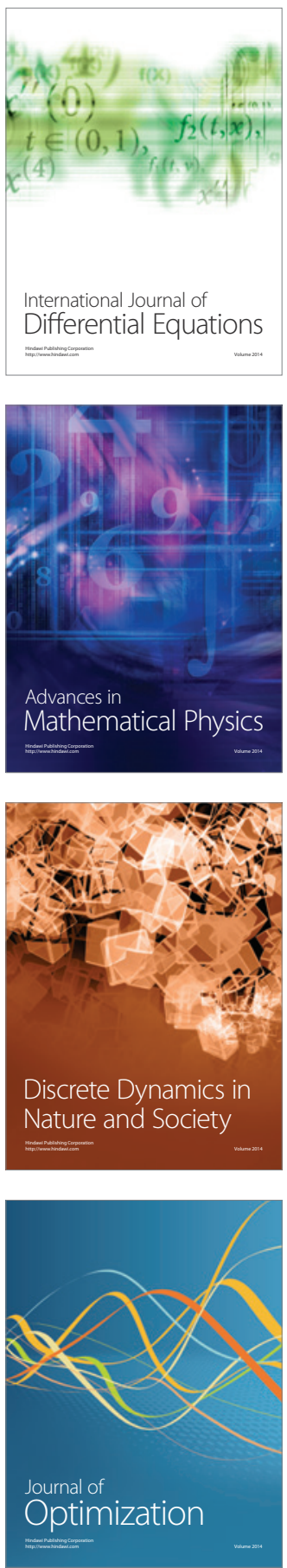